\title{
Quantitative Trait Loci for High-Temperature Adult-Plant and Slow-Rusting Resistance to Puccinia striiformis f. sp. tritici in Wheat Cultivars
}

\author{
Q. Guo, Z. J. Zhang, Y. B. Xu, G. H. Li, J. Feng, and Y. Zhou
}

First, second, third, fifth, and sixth authors: Department of Plant Pathology, China Agricultural University, Beijing 100094, China; and fourth author: College of Science, China Agricultural University, Beijing 100094, China.

Accepted for publication 27 December 2007.

\begin{abstract}
Guo, Q., Zhang, Z. J., Xu, Y. B., Li, G. H., Feng, J., and Zhou, Y. 2008. Quantitative trait loci for high-temperature adult-plant and slow-rusting resistance to Puccinia striiformis f. sp. tritici in wheat cultivars. Phytopathology 98:803-809.

Stripe rust, caused by Puccinia striiformis f. sp. tritici, is one of the most damaging diseases of wheat (Triticum aestivum) globally. High-temperature adult-plant resistance (HTAPR) and slow-rusting have great potential for sustainable management of the disease. The wheat cultivars Luke and Aquileja have been previously reported to possess HTAPR and slow-rusting to stripe rust, respectively. Aquileja displayed less number of stripes per unit leaf area than Luke, while Luke showed lower infection type than Aquileja at adult-plant stages of growth under high-temperature conditions. The objectives of this study were to confirm the resistances and to map the resistance genes in Luke and Aquileja. Luke was crossed with Aquileja, and 326 of the $F_{2}$ plants were genotyped using 282 microsatellite primer pairs. These $F_{2}$ plants and their derived $F_{3}$ families

adult plants only. Two quantitative trait loci (QTL) were identified, on the short arm of chromosome $2 \mathrm{~B}$, to be significantly associated with infection type at adult-plant stages in the fields and in the high-temperature greenhouse. The locus distal to centromere, referred to as QYrlu.cau-2BS1, and the locus proximal to centromere, referred to as QYrlu.cau-2BS2, were separated by a genetic distance of about $23 \mathrm{cM}$. QYrlu.cau-2BS1 was flanked by the microsatellite markers Xwmc154 and Xgwm148, and QYrlu.cau-2BS2 was flanked by Xgwm148 and Xabrc167. QYrlu.cau$2 B S 1$ and QYrlu.cau-2BS2 explained up to 36.6 and $41.5 \%$ of the phenotypic variation of infection type, respectively, and up to $78.1 \%$ collectively. No significant interaction between the two loci was detected. Another QTL, referred to as QYraq.cau- $2 B L$, was detected on the long arm of chromosome $2 \mathrm{~B}$ to be significantly associated with stripe number. QYraq.cau- $2 B L$ was flanked by the microsatellite markers Xwmc175 and $X w m c 332$, and it explained up to $61.5 \%$ of the phenotypic variation of stripe number. It is possible that these three QTL are previously unmapped loci for resistance to stripe rust.
\end{abstract} were evaluated for resistance to stripe rust by inoculation in the fields and greenhouses of high- and low-temperatures. Infection type was recorded for both seedlings and adult plants, and stripe number was recorded for
Additional keywords: durable resistance, simple sequence repeat, yellow rust.
Stripe (yellow) rust is a major disease of wheat globally (25). It is the most destructive plant disease in some regions of the world such as the Pacific Northwest of the United States and the Northwest of China $(12,27)$. Use of host resistance is an important strategy to manage the disease. More than 40 genes conferring race-specific resistance to stripe rust have been documented (15), but most of them have been overcome by new virulent races of Puccinia striiformis Westend. f. sp. tritici Eriks. (12,27). In contrast, some wheat cultivars displayed non-race-specific and durable resistance to $P$. striiformis f. sp. tritici, among which are the well-reported Luke (17), Alpowa (11), Camp Remy (14), and Aquileja (7,29). The resistance in these cultivars exhibits in quantitative fashions, being different from the race-specific resistance which primarily exhibits in hypersensitive reaction or in qualitative fashions. Two types of quantitative resistance, i.e., hightemperature adult-plant resistance (HTAPR) and slow-rusting resistance, have been intensively investigated $(9,12)$. As described by Line (12), seedlings of plants with HTAPR are susceptible (high infection type) at both low $\left(6\right.$ to $21^{\circ} \mathrm{C}$ ) and high (13 to $32^{\circ} \mathrm{C}$ ) temperatures, and adult plants are susceptible at low temperatures but resistant (low infection type) at high temperatures in greenhouse. In the field, expression of HTAPR begins at stem

Corresponding author: Z. J. Zhang; E-mail address: zhangzj@ cau.edu.cn

doi:10.1094/PHYTO-98-7-0803

(C) 2008 The American Phytopathological Society elongation and becomes stronger at later stages of growth. In the Pacific Northwest of the United States, HTAPR has consistently proven to be durable against all $P$. striiformis f. sp. tritici races. For more than 40 years, there has been no evidence of race specificity for HTAPR in wheat (12). As described by Caldwell (5), slow-rusting resistance can be characterized by slow disease development in the field despite a susceptible infection type, and by the presence of one or more resistance components, such as longer latent period, lower receptivity or infection frequency, smaller uredium size, and reduced spore production. Slow-rusting has been identified for wheat $P$. striiformis f. sp. tritici (9). Despite the observations that some initially slow-rusting wheat cultivars were proven to show race specificity for $P$. striiformis f. sp. tritici, the $P$. striiformis f. sp. tritici slow-rusting in some other wheat cultivars such as Cappelle Desprez was reported to be nonrace-specific and durable (9). The non-race specificity and durability of the HTAPR and the slow-rusting confer upon these two types of resistance great potential for sustainable management of $P$. striiformis f. sp. tritici disease $(9,12)$. HTAPR and slow-rusting are generally inherited in a quantitative fashion $(9,12)$. DNA marker technology constitutes a powerful tool for studying the genetics of quantitative traits, and it has been applied to identifying quantitative trait loci (QTL) conferring $P$. striiformis f. sp. tritici resistance in Camp Remy (14) and Alpowa (11).

Luke was developed by O. Vogel and colleagues at the USDAARS, Pullman, Washington with a high level of HTAPR to $P$. striiformis f. sp. tritici. The cultivar was widely grown in the 
Pacific Northwest of the United States in the 1970s to 1990s and is still widely used in wheat breeding programs in the region as an important genetic stock for the durable type resistance $(12,17)$. The HTAPR in Luke is characterized by a range of infection types depending upon temperature and stage of plant growth as described above $(12,17)$. For more than 30 years, there has been no evidence of race specificity for the HTAPR in Luke (12).

Aquileja has been widely grown for more than 25 years in the Northwest of China where new virulent $P$. striiformis f. sp. tritici races or isolates frequently occurred during this period, but it still keeps its slow-rusting without showing detectable race specificity $(7,29)$. The slow-rusting in Aquileja is primarily characterized by a reduction in the penetration of $P$. striiformis f. sp. tritici $(7,8)$. The number of sub-stomatal vesicle and the number of stripe per unit leaf area were less in Aquileja than in Luke, but Aquileja showed higher infection type than Luke at adult-plant stages under the high temperature conditions (7). Despite the practical value of the two cultivars, genes conferring the P. striiformis $\mathrm{f}$. sp. tritici resistance in Luke and Aquileja have not been mapped.

The objectives of this study were to confirm the HTAPR in Luke and the slow-rusting in Aquileja, and to map the QTL conferring infection type and stripe number in the cross of Luke/ Aquileja under the field and greenhouse conditions.

\section{MATERIALS AND METHODS}

Field evaluation of resistance. The wheat germplasm used were Aquileja (PI 393993) and Luke (CItr 14586), and their $F_{1}$, $\mathrm{F}_{2}$, and $\mathrm{F}_{3}$. Three hundred and twenty-six $\mathrm{F}_{2}$ plants which were derived from two individual $F_{1}$ plants (134 from one and 192 from the other), and the $F_{3}$ families descended from these $F_{2}$ plants were used to construct the genetic map and to detect QTL. The wheat cultivar Ming Xian 169 (ZM 009379) was used as the susceptible check (hereafter referred to as MX). MX is highly susceptible to all known $P$. striiformis f. sp. tritici races in China (27).

Field experiments were conducted at the China Agricultural University farms in Beijing where the wheat cropping season lasts from the early-October to the following early-July. The monthly mean minimum, mean maximum, and mean temperatures were presented in Table 1 for the months in which the weather conditions are favorable to $P$. striiformis f. sp. tritici in the Beijing region. These data indicate that the temperatures in the late-springs and early-summers are suitable for the expression of the HTAPR of Luke.
During the 2004 to 2005 cropping season, parents, $F_{1}, F_{2}$, and MX were sown in rows $2 \mathrm{~m}$ long with 10 seeds in each row and with $30 \mathrm{~cm}$ of space between adjacent rows. The experiment consisted of 34 rows of $\mathrm{F}_{2}$, and 4 rows of each parent, $\mathrm{F}_{1}$, and $\mathrm{MX}$. During the 2005 to 2006 cropping season, the $F_{3}$ families derived from the $134 \mathrm{~F}_{2}$ plants as specified above, together with the parents, $\mathrm{F}_{1}$, and $\mathrm{MX}$, were sown in a randomized complete-block design with 3 replicates, in rows $1 \mathrm{~m}$ long with 10 seeds in each row for $\mathrm{F}_{3}$ and 5 seeds in each row for the other genotypes, and with $30 \mathrm{~cm}$ of space between adjacent rows. Ten seeds of each $\mathrm{F}_{3}$ family were sown in each replicate. During the 2006 to 2007 cropping season, the $F_{3}$ families derived from the $192 F_{2}$ plants, and the parents, $\mathrm{F}_{1}$, and $\mathrm{MX}$, were grown in the same way in the 2005 to 2006 season.

For each of the three test seasons, the plants were inoculated with a single spore-derived isolate of the Chinese race CYR32 of $P$. striiformis f. sp. tritici. CYR32 is virulent to the resistance genes $Y r 1, Y r 2, Y r 3, Y r 4, Y r 6, Y r 7, Y r 9, Y r 17, Y r 22, Y r 23, Y r 27$, $Y r A, Y r C V 1, Y r C V 2, Y r C V 3, Y r G, Y r S D$, and $Y r S O$ (27). Although the weather conditions are favorable to $P$. striiformis f. sp. tritici in the northern China including Beijing region from the early spring to the early summer, $P$. striiformis f. sp. tritici is unable to oversummer in this region (4) and thus it does not frequently occur naturally in the Beijing region (27). Artificial inoculation is necessary to ensure adequate stripe rust in the experimental fields. In fact, $P$. striiformis f. sp. tritici was not observed in wheat production fields from 2003 to 2007, during which this study was conducted, and no natural inoculum contaminated the experiments.

For the seedling tests, the inoculation was conducted when the seedlings were recovering from the winters (GS 18 to 20 on Zadoks scale [28]). The inoculation date was in mid-March in all three test years. Pathogen multiplication, inoculation, and incubation were done as described previously (8). Five to seven days after sporulation started, three tillers of each plant were evaluated on the most seriously-diseased leaf of each tiller for infection type (IT) on the 0 to 9 scale (13). The number of plants evaluated was 326 for $\mathrm{F}_{2}, 24$ to 30 for each $\mathrm{F}_{3}$ family, 29 to 37 (during the 2004 to 2005 season) and 48 to 60 (during the 2005 to 2006 and 2006 to 2007 seasons) for each parent, $\mathrm{F}_{1}$, and $\mathrm{MX}$.

For the adult-plant tests, the inoculation was conducted when flag leaves unfolded completely (GS 39 to 45 on Zadoks scale [28]). The plants for the adult stage tests were the same ones as tested at the seedling stage since the seed of some $\mathrm{F}_{3}$ families was not adequate for separate seedling and adult-plant tests. The aim

TABLE 1. The monthly mean minimum, mean maximum, and mean temperature for the months in which the weather conditions are favorable to Puccinia striiformis f. sp. tritici at the China Agricultural University farms where the field experiments were conducted, and the wheat growth stages approximately corresponding to each of the months from 2005 to 2007

\begin{tabular}{|c|c|c|c|c|}
\hline \multirow[b]{2}{*}{ Month } & \multirow[b]{2}{*}{ Stage } & \multicolumn{3}{|c|}{ Year } \\
\hline & & 2005 & 2006 & 2007 \\
\hline \multirow[t]{2}{*}{ March } & Seedling growth (GS 17 to 19$)$ & & & \\
\hline & $\mathrm{M}^{\mathrm{b}}$ & 6.0 & 8.2 & 5.9 \\
\hline \multirow[t]{2}{*}{ April } & Seedling growth to tillering (GS 19 to 29 ) & & & \\
\hline & Min & 10.0 & 8.2 & 9.2 \\
\hline \multirow[t]{4}{*}{ May } & Stem elongation to anthesis (GS 30 to 69 ) & & & \\
\hline & Min & 14.4 & 15.4 & 16.9 \\
\hline & Max & 25.2 & 26.0 & 28.6 \\
\hline & M & 19.8 & 20.7 & 22.7 \\
\hline \multirow[t]{3}{*}{ June } & Milk to hard dough (GS 70 to 89 ) & & & \\
\hline & Min & 20.8 & 20.7 & 21.7 \\
\hline & Max & 31.8 & 31.4 & 32.2 \\
\hline
\end{tabular}

${ }^{a}$ GS = growth stage on Zadoks scale.

${ }^{\mathrm{b}} \mathrm{Min}=$ mean minimum temperature, Max = mean maximum temperature, and $\mathrm{M}=$ mean temperature. The temperature was in degree Celsius. 
of the inoculation at the adult-plant stage was to enhance the infection for evaluating the disease reaction of flag leaves. All inoculations were made in the early- to mid-May. Six to eight days after symptoms appeared, three flag leaves of each plant were evaluated for the number of stripes as well as for IT. The length and width of the middle of each flag leaf were measured, and the number of stripes per $10 \mathrm{~cm}^{2}$ leaf area (SN) was taken as a measurement on a resistance component of the slow-rusting.

Greenhouse evaluation of resistance. Parents, $F_{1}, 134 \mathrm{~F}_{3}$ families, and MX were sown on November 1, 2006 in two P. striiformis f. sp. tritici-free greenhouses, one each for the high and low temperature conditions. A randomized complete-block design with 3 replicates was used. The sowing was done in plastic pots
(30 $\mathrm{cm}$ in diameter and $25 \mathrm{~cm}$ in height) with 5 seeds per pot. In each greenhouse, 30 seeds of each $\mathrm{F}_{3}$ family were sown in the 3 replicates with 10 seeds per replicate. Each replicate consisted of 2 pots per $\mathrm{F}_{3}$ family, 6 pots of each parent, $\mathrm{F}_{1}$, and MX. After sowing, the greenhouses were operated with the temperature regime of 6 to $21^{\circ} \mathrm{C}$. The diurnal temperatures were programmed to change gradually between the minimum at 2:00 a.m. during the 8-h dark period and the maximum at 2:00 p.m. during the $16-\mathrm{h}$ light period (17), and this operation program was also applied to the other temperature regimes as shown below.

For the seedling test, inoculation was done on 14 November 2006 when the plants were at the two-leaf stage (GS 12 on Zadoks scale [28]). The inoculated plants were incubated at 6 to
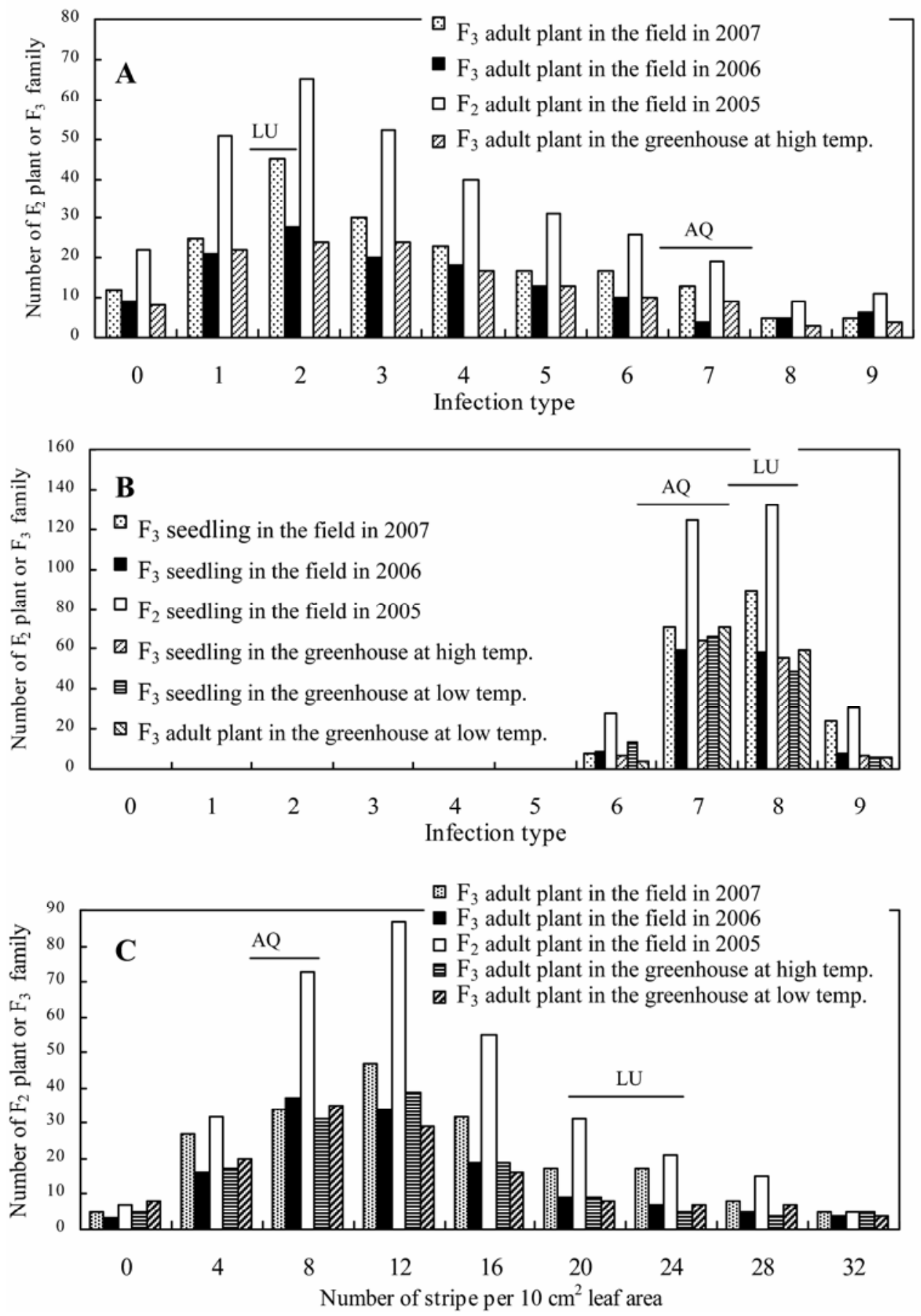

Fig. 1. Frequency distributions of $\mathrm{F}_{2}$ plants and $\mathrm{F}_{3}$ families of the wheat cross Luke/Aquileja for infection type for the experiments in which the high-temperature adult-plant resistance was expressed (A) and for the experiments in which the high-temperature adult-plant resistance was not expressed (B), and for the stripe number per $10 \mathrm{~cm}^{2}$ leaf area at adult-plant stage $(\mathbf{C})$, tested with Puccinia striiformis $\mathrm{f}$. sp. tritici under field and greenhouse conditions. LU $=\mathrm{Luke}$ and $\mathrm{AQ}=$ Aquileja. The horizontal bars indicate the range of disease values of the parents LU and AQ. 
$10^{\circ} \mathrm{C}$ for $24 \mathrm{~h}$, and then one of the greenhouses was kept at the low temperature regime of 6 to $21^{\circ} \mathrm{C}$, while the other was operated at the high temperature regime of 13 to $32^{\circ} \mathrm{C}$. The IT was evaluated using the 0 to 9 scale (13) for each plant 6 to 8 days after sporulation started. The number of plants evaluated was 27 to 30 for each $F_{3}$ family, and 60 for each parent, $F_{1}$, and $\mathrm{MX}$ in each greenhouse.

From 20 December 2006 to the end of February, 2007, the greenhouses were kept unheated (about -2 to $8^{\circ} \mathrm{C}$ ) to vernalize the plants. Beginning from 1 March, the greenhouses were operated at 6 to $21^{\circ} \mathrm{C}$.

For the adult-plant test, the inoculation was conducted on 16 April when the flag leaves unfolded completely (GS 39 to 45 on Zadoks scale [28]). The inoculated plants were incubated at 6 to $10^{\circ} \mathrm{C}$ for $24 \mathrm{~h}$, and then one of the greenhouses was kept at the low temperature regime of 6 to $21^{\circ} \mathrm{C}$, while the other was operated at the high temperature regime of 13 to $32^{\circ} \mathrm{C}$. Three flag leaves of each plant were evaluated for SN and IT, as described previously, 6 to 8 days after symptoms appeared.

Detection of QTL for the resistance. Fresh leaves from the parents, $F_{1}$, and $F_{2}$ plants grown in 2004 to 2005 were ground in liquid nitrogen. DNA was extracted from the leaf powder using the cetyl trimethyl ammonium bromide (CTAB) method (19). Microsatellite simple sequence repeat (SSR) analysis was conducted following Röder et al. (20). Polymerase chain reaction (PCR) reactions were performed in a Perkin Elmer Cetus DNA thermal cycler 480 and a GeneAmp PCR System 9700. PCR products were separated in $6 \%$ denaturing polyacrylamide gels and then visualized using the silver staining method (1). The sequences of SSR primers were acquired from the published data including GWM (Gatersleben wheat microsatellite [20]), GDM (Gatersleben D genome microsatellites [16]), WMC (Wheat Microsatellite Consortium, P. Isaac, IDnagenetics, Norwich, UK),
BARC (Beltsville Agriculture Research Center [24]), and CFA (Clermont-Ferrand A genome), and CFD (Clermont-Ferrand D genome, P. Sourdille, INRA).

Of the $980 \mathrm{SSR}$ primer pairs used to screen parents and $\mathrm{F}_{1}, 282$ were polymorphic between the parents Luke and Aquileja. The polymorphic markers were then used for genotyping the $326 \mathrm{~F}_{2}$ plants. The marker loci that distorted from 1:2:1, 1:3, or 3:1 $(P<$ 0.05 for chi-square test) were discarded. Linkage analysis was conducted using the software MAPMAKER/EXP 3.0 (10) set to Kosambi mapping function in centimorgan (cM) with a logarithm of the odds (LOD) threshold of 6.0, and the other mapping parameters and procedures were similar to those as reported previously (14). A map was constructed containing 229 marker loci that were distributed over the 21 chromosomes with 6 to 16 loci on each chromosome. Linkage groups were assigned by referring to the ITMI (International Triticae Mapping Initiative) maps $(20,23)$.

QTL analysis was conducted on the data of IT and SN using composite interval mapping (CIM) and multiple interval mapping (MIM) in Windows QTL Cartographer 2.0 (2). For CIM, the threshold LOD score to declare significance at $\alpha=0.05$ was 3.4 to 3.6 for IT and 3.6 to 4.0 for SN as estimated empirically with 1,000 permutations by using the facilities in the Cartographer. The other parameters and procedures of the analysis were similar to those as reported previously (14). For MIM analysis, the QTL peaks with an LOD score threshold value of 3.4 from the CIM analysis were used as the initial model. New QTL was added to the current model in an iterative and stepwise fashion. The proportion of phenotypic variation explained by individual QTL and by the whole model (total $R^{2}$ ), the additive and dominant effect, and the QTL $\times$ QTL interaction were estimated using the 'summary' option of MIM.

The data processing, chi-square test, and Kolmogorov-Smirnov tests for normality were done using the SAS software (22).

TABLE 2. Mean \pm standard error of the parental cultivars Aquileja and Luke, $F_{1}$, and the susceptible check wheat cultivar Ming Xian 169 for their infection type and the number of stripe per $10 \mathrm{~cm}^{2}$ leaf area, tested with Puccinia striiformis f. sp. tritici under the field and greenhouse conditions

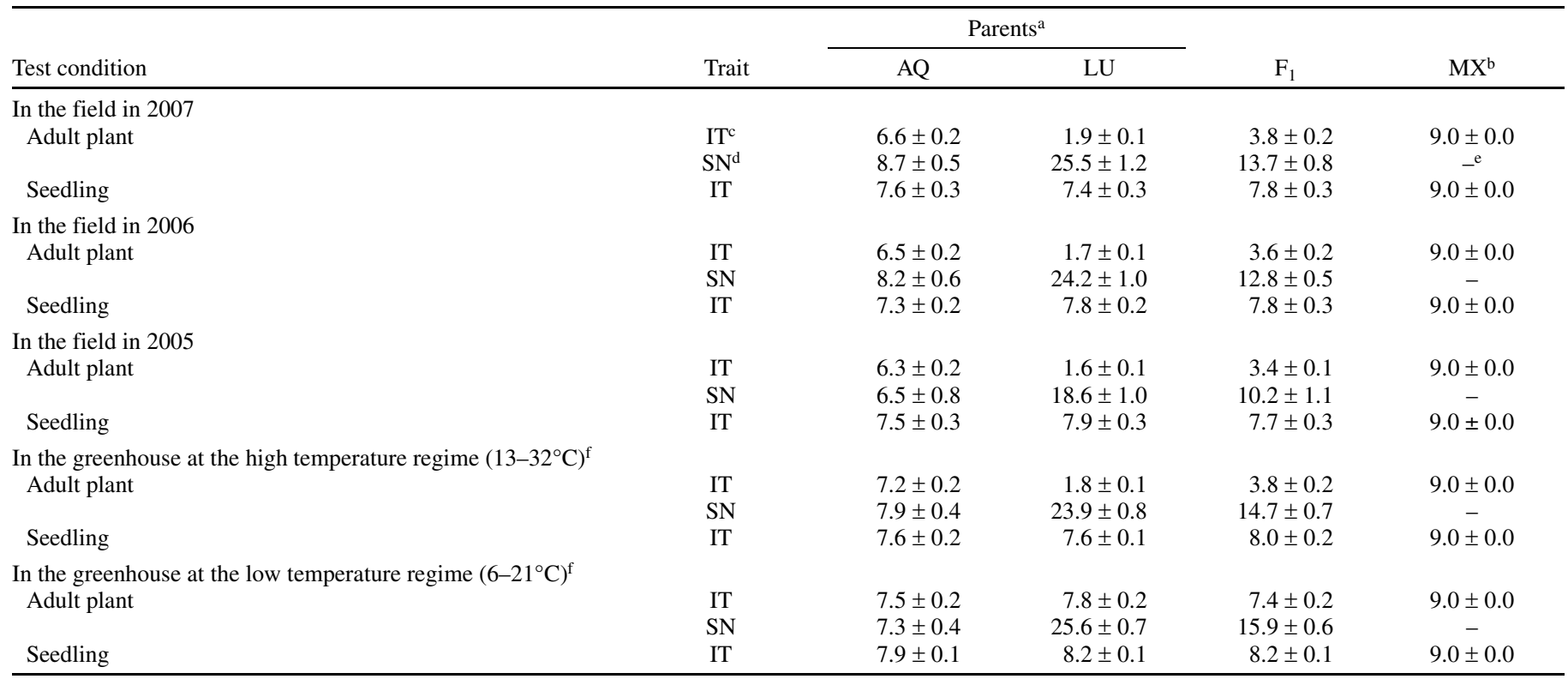

a $\mathrm{AQ}=$ Aquileja and $\mathrm{LU}=$ Luke.

${ }^{\mathrm{b}} \mathrm{MX}=$ the susceptible check wheat cultivar Ming Xian 169.

${ }^{\mathrm{c}}$ IT $=$ infection type. IT was recorded on the 0 to 9 scale. This scale has been described by Line et al.: $0=$ no visible signs or symptoms; $1=$ necrotic or chlorotic flecks with no sporulation; $2=$ necrotic and/or chlorotic blotches or stripes with no sporulation; $3=$ necrotic and/or chlorotic blotches or stripes with only a trace of sporulation; 4,5 , and $6=$ necrotic and/or chlorotic blotches or stripes with light, intermediate, and moderate sporulation; respectively; and 7, 8 , and $9=$ abundant sporulation with necrotic and/or chlorotic blotches or stripes, chlorosis behind the sporulation area, and no chlorosis or necrosis, respectively. Blotches occur on seedlings and stripes occur on adult plants.

${ }^{\mathrm{d}} \mathrm{SN}=$ stripe number per $10 \mathrm{~cm}^{2}$ leaf area.

${ }^{\mathrm{e}}$ On the susceptible check MX, individual stripes were apt to coalesce and were not very distinct, and thus the SN data were not presented.

${ }^{\mathrm{f}}$ Temperatures were programmed to change gradually between the high extreme at 2:00 p.m. during the 16-h light period and the low extreme at 2:00 am during the 8-h dark period. 


\section{RESULTS AND DISCUSSION}

HTAPR and slow-rusting resistance in the cross Luke/ Aquileja. The disease data were presented in Table 2 and Figure 1 for adult plants for both IT and SN, and for seedlings for IT only. Individual lesions of $P$. striiformis $\mathrm{f}$. sp. tritici on seedlings were apt to coalesce and were not very visually distinct, thus the number of lesions per $10 \mathrm{~cm}^{2}$ leaf area was not given for seedlings. The susceptible check MX displayed the highest IT of nine in every experiment, indicating that conditions were adequate for disease evaluation. Several points can be seen from Table 2 and Figure 1. First, Luke showed low IT at adult-plant stages in the fields and in the greenhouse of high temperature regime, confirming the expression of the HTAPR in Luke as previously reported by other authors $(12,17)$. Second, Aquileja displayed a lower value of SN than Luke at the adult-plant stage, confirming the major resistance component of the slow-rusting in Aquileja as previously reported $(7,8,29)$. Unfortunately, this study presents no data of lesion number for seedling stage. However, our previous tests showed that Aquileja displayed less numbers of substomatal vesicles than Luke by at least twofold at seedling stage (7), meaning that a slow-rusting component (i.e., reduction in penetration) was expressed at the seedling stage of Aquileja. Third, Aquileja showed higher IT than Luke in the experiments where the HTAPR was expressed. Fourth, SN was not substantially influenced by the low $\left(6\right.$ to $\left.21^{\circ} \mathrm{C}\right)$ and high $\left(13\right.$ to $\left.32^{\circ} \mathrm{C}\right)$ temperatures for Aquileja, Luke, and their $F_{1}$. This can be evidenced by the fact that no significant difference in $\mathrm{SN}$ was found between the low and high temperature greenhouse experiments by the $t$ tests conducted by using the means and standard errors in Table 2. Lastly, the SN of $\mathrm{F}_{1}$ was between those of the parents Luke and Aquileja in all experiments, and the IT of $F_{1}$ was between those of Luke and Aquileja in the experiments where the HTAPR was expressed. The distributions of $F_{2}$ plants and $F_{3}$ families were continuous and unimodal for both IT (Fig. 1A) and SN (Fig. 1C). These results indicate that both IT and SN were inherited in a quantitative fashion. The Kolmogorov-Smirnov tests did not reject $(D<$ $0.082, P>0.05)$ the normality of the distributions. In addition, it can be noticed that the range of IT distribution was from 0 to 9 in the experiments where the HTAPR was expressed (Fig. 1A), contrasting the range from 6 to 9 in the experiments where the HTAPR was not expressed (Fig. 1B).

QTL analysis. Two QTL were identified, on the short arm of chromosome $2 \mathrm{~B}$, to be significantly associated with IT at adultplant stages in the fields (Fig. 2A) and in the high-temperature greenhouse (Fig. 2B). The locus distal to centromere is referred to as QYrlu.cau-2BS1 and the locus proximal to centromere as QYrlu.cau-2BS2. QYrlu.cau-2BS1 is flanked by the SSR markers $X w m c 154$ and Xgwm148, and QYrlu.cau-2BS2 is flanked by Xgwm148 and Xabrc167. The two loci are separated by a genetic distance of about $23 \mathrm{cM}$. The position of the LOD score peak at each of the two loci is consistent among the 4 HTAPR-expressed experiments ( 3 in the fields, Fig. 2A, and 1 in the high-temperature greenhouse, Fig. 2B). Multiple interval mapping indicated that QYrlu.cau-2BS1 and QYrlu.cau-2BS2 reduced IT by up to 1.8 and 2.1 in an additive fashion, and explained up to 36.6 and $41.5 \%$ of the phenotypic variation of IT, respectively, and up to $78.1 \%$ collectively. Dominant and epistatic effects were not significant and thus not presented. These data (Fig. 2A and B) also showed that both QYrlu.cau-2BS1 and QYrlu.cau-2BS2 have no substantial effect on IT at seedling stages in both the fields and the greenhouses, and at adult-plant stages in the low-temperature greenhouse either. At both loci, Luke contributed the QTL that reduce IT (Table 3). The two QTL for HTAPR detected here differ in chromosome location from the other HTAPR genes or QTL previously mapped $(11,21,26)$. Santra et al. (21) reported an HTAPR QTL in the wheat cultivar Stephens on chromosome arm 6BS. On the same chromosome region of the Stephens QTL,
Uauy et al. (26) mapped the HTAPR gene Yr36. Lin and Chen (11) mapped the HTAPR QTL Yr39 on the chromosome 7BL. In the chromosome regions corresponding to the QYrlu.cau-2BS1 and QYrlu.cau-2BS2 locations, QTL for $P$. striiformis f. sp. tritici resistance have been identified from the wheat cultivars Opata 85 (3), Kariega (18), and Camp Remy (14). Luke shares no commonality in ancestry with these three cultivars. It is possible that QYrlu.cau-2BS1 and QYrlu.cau-2BS2 are two previously unmapped loci for HTAPR.
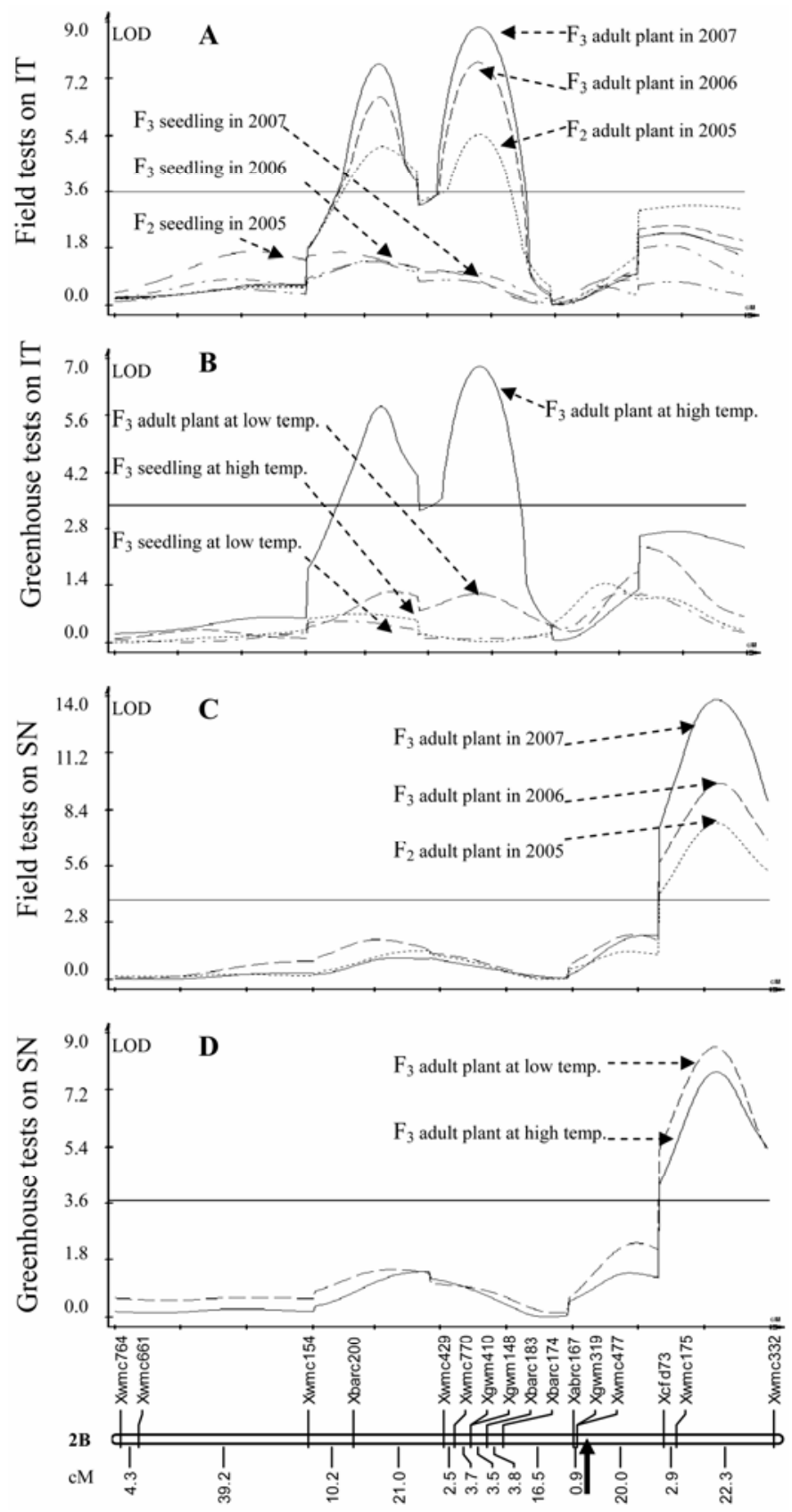

Fig. 2. Logarithm of the odds curves for the quantitative trait loci mapped on the short arm of chromosome $2 \mathrm{~B}$ that reduced infection type under the field conditions (A) and under the greenhouse conditions (B), and on the long arm of chromosome $2 \mathrm{~B}$ that reduced the stripe number per $10 \mathrm{~cm}^{2}$ leaf area under the field conditions (C) and under the greenhouse conditions (D) in the wheat cross Luke/Aquileja, tested with Puccinia striiformis f. sp. tritici. Short arm is toward the left. Solid arrow indicates the approximate position of centromere. The positions of the microsatellite (or simple sequence repeat, SSR) markers are shown along the chromosome. $\mathrm{cM}=$ centimorgan, IT = infection type, $\mathrm{SN}=$ stripe number per $10 \mathrm{~cm}^{2}$ leaf area, and LOD $=$ logarithm of the odds. The horizontal line indicates the LOD significance threshold. 
Another QTL referred to as QYraq.cau-2BL was detected, on the long arm of chromosome $2 \mathrm{~B}$, to be significantly associated with SN at adult-plant stages in the fields (Fig. 2C) and in the greenhouses as well (Fig. 2D). QYraq.cau-2BL is flanked by the SSR markers Xwmc175 and Xwmc332. The position of the LOD score peak at QYraq.cau- $2 B L$ is consistent among the five experiments ( 3 in the fields, Fig. 2C, and 2 in the greenhouses, Fig. 2D). This QTL explained up to $61.5 \%$ of the phenotypic variation of $\mathrm{SN}$ and the resistance gene came from Aquileja (Table 3). The resistance conferred by QYraq.cau- $2 B L$ could be expressed in both high- and low-temperature tests (Fig. 2D). QTL for slowrusting have been reported previously $(6,14)$ in the chromosome region corresponding to the QYraq.cau-2BL location. In those reports, the resistance was derived from the wheat cultivars Deben (6), and Camp Remy which has been implicated to originate from Cappelle Desprez (14). Pedigree analyses did not show that Aquileja shares common ancestor with any of the three cultivars. The possibility exists that QYraq.cau- $2 B L$ is a previously unmapped $P$. striiformis $\mathrm{f}$. sp. tritici-resistance QTL.

It can be noticed, as shown in Figure $2 \mathrm{~A}$ and $\mathrm{B}$, that there are some LOD peaks near the SSR marker Xwmc175 on the chromosome arm 2BL. These peaks did not reach the LOD thresholds, but they consistently occurred in the same chromosome region for the experiments conducted under different conditions. The consistency may suggest that some QTL for IT exists in this region. Different from QYrlu.cau-2BS1 and QYrlu.cau-2BS2 where the resistance was contributed by Luke, in the Xwmc175 region the resistance originated from Aquileja. Interestingly, these peaks occurred in the same chromosome region of QYraq.cau-2BL. It can be assumed that the QYraq.cau- $2 B L$ was responsible for the reduction of IT to some extent (Fig. 2A and B) as well as for the reduction of SN (Fig. 2C and D).

Other loci may also contribute to the resistance in the cross Luke/Aquileja, but they were not detected because the map pre- sented here was only a partial one. Considering the fact that up to $78.1 \%$ of the IT variation could be explained by QYrlu.cau-2BSI and QYrlu.cau-2BS2, it is unlikely that additional QTL of large effect can be found in the remaining genome for IT. For SN, however, only one QTL, i.e., QYraq.cau- $2 B L$, was identified that accounted for 47.0 to $61.5 \%$ of the $\mathrm{SN}$ variation. It can be assumed that some QTL for SN of less effect than QYraq.cau-2BL may exist but not be detected, and such QTL should be responsible for at least $38.5 \%$ of the $\mathrm{SN}$ variation. A reason for failing to detect such SN QTL might be that the experiments for SN were conducted with an accuracy that was not high enough for the detection. This would be understandable when considering that the same plants were inoculated twice. In general cases of susceptible plants, the $P$. striiformis f. sp. tritici inoculated on seedlings can produce enough spores to reinfect the upper leaves of the plants at adult-plant growth stages. However, the plants in the present study were derived from the resistant/resistant cross of Aquileja with Luke. After stem elongation in the fields and in the hightemperature greenhouse as described above, most of the plants had an IT less than 5 on the 0 to 9 scale, indicating that the amount of spores produced from these plants was considerably limited. In order to decrease the chance for a plant to escape from inoculum and to enhance the pressure and uniformity of the inoculum, the second inoculation was conducted when flag leaves unfolded completely. The continued sporulation of the P. striiformis f. sp. tritici resulted from the first inoculation on the lower leaves, might interfere with the second inoculation, and thus decrease the accuracy of the counting of $\mathrm{SN}$. The inadequate accuracy might affect the detection of some SN QTL of minor effect. Luckily, the interference, if any, seemed not to substantially influence the identification of the QYraq.cau- $2 B$ since this QTL was consistently detected at the same chromosome position in each of the five experiments as shown in Figure 2C and D. We assume that the large effect of QYraq.cau- $2 B$ on $\mathrm{SN}$ considerably outweighed the interference.

TABLE 3. Quantitative trait loci, that are related to the reduction of infection type or stripe number per $10 \mathrm{~cm}^{2}$ leaf area caused by Puccinia striiformis f. sp. tritici, detected in the wheat cross Luke/Aquileja under the field and greenhouse conditions

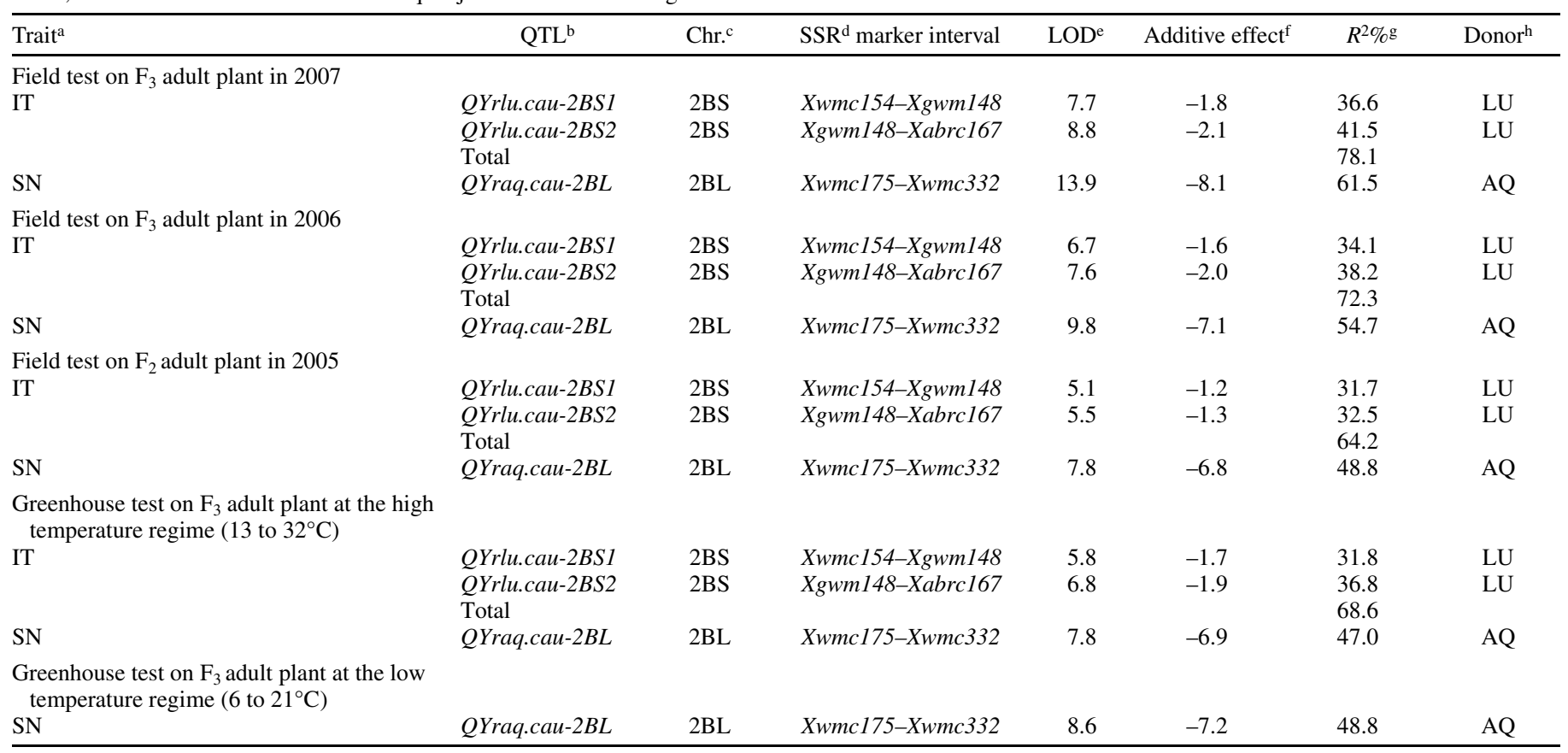

${ }^{a}$ IT $=$ infection type and $\mathrm{SN}=$ stripe number per $10 \mathrm{~cm}^{2}$ leaf area. IT was recorded on the 0 to 9 scale.

${ }^{\mathrm{b}} \mathrm{QTL}=$ quantitative trait locus.

${ }^{\mathrm{c}} \mathrm{Chr}$ = chromosome arm where the QTL locates.

${ }^{\mathrm{d}} \mathrm{SSR}=$ simple sequence repeat or microsatellite.

${ }^{\mathrm{e}} \mathrm{LOD}=$ logarithm of the odds. The LOD value was estimated by the composite interval mapping.

${ }^{\mathrm{f}}$ The effect was estimated by the multiple interval mapping.

$\mathrm{g}$ The percentage of phenotypic variation explained by the QTL was estimated by the multiple interval mapping.

${ }^{\mathrm{h}}$ The parent that contributed the marker interval or QTL that reduced IT or SN. LU $=$ Luke, and AQ = Aquileja. 


\section{ACKNOWLEDGMENTS}

We thank the anonymous reviewers for their valuable comments on the manuscript. This study was supported by the National Natural Science Foundation of China (Grant Nos. 30270905 and 30370920) and the National "973" Project of China (Grant No. 2006CB101901).

\section{LITERATURE CITED}

1. Bassam, B. J., Caetano-Anolles, G., and Gresshoff, P. M. 1991. Fast and sensitive silver staining of DNA in polyacrylamide gels. Anal. Biochem. 196:80-83.

2. Basten, J. C., Wang, S., Gaffney, P., and Zeng, Z. B. 2003. Windows QTL Cartographer, Version 2.0, Statistical Genetics, North Carolina State University, Raleigh, NC.

3. Börner, A., Schumann, E., Fürste, A., Cöster, H., Leithold, B., Rörder, M. S., and Weber, W. E. 2002. Mapping of quantitative trait loci determining agronomic important characters in hexaploid wheat (Triticum aestivum L.). Theor. Appl. Genet. 105:921-936.

4. Brown, J. K. M., and Hovmøller, M. S. 2002. Aerial dispersal of pathogen on the global and continental scales and its impact on plant disease. Science 297:537-541.

5. Caldwell, R. M. 1968. Breeding for general and/or specific plant disease resistance. Pages 263-272 in: Proc. Intl. Wheat Genet. Symp., 3rd. K. W. Finlay and K. W. Shepherd, eds. Austr. Acad. Sci., Canberra, Australia.

6. Christiansen, M. J., Feenstra, B., Skovgaard, I. M., and Andersen, S. B. 2006. Genetic analysis of resistance to yellow rust in hexaploid wheat using a mixture model for multiple crosses. Theor. Appl. Genet. 112:581591.

7. Feng, J., Zhang, Z. J., Li, G., Zhou, Y., Wang, H., Guo, Q., and Sun, J. 2007. Components of quantitative, durable resistance to stripe rust in five wheat cultivars and the genetic distance among the cultivars. Acta Phytopathol. Sin. 37:175-183.

8. Feng, J., Zhang, Z. J., Li, G., Zhou, Y., and Guo, Q. 2007. Relationships and genetics of wheat effects on infection frequency and colony extension of Puccinia striiformis f. sp. tritici. Eur. J. Plant Pathol. 120:223-232.

9. Johnson, R. 1992. Reflection of a plant pathologist on breeding for disease resistance, with emphasis on yellow rust and eyespot of wheat. Plant Pathol. 41:239-254.

10. Lander, E. S., Green, P., Abrahamson, J., Barlow, A., Daly, M. J., Lincoln, S. E., and Newburg, L. 1987. MAPMAKER: An interactive computer package for constructing primary genetic linkage maps of experimental and natural populations. Genomics 1:174-181.

11. Lin, F., and Chen, X. M. 2007. Genetics and molecular mapping of genes for race-specific all-stage resistance and non-race-specific high-temperature adult-plant resistance to stripe rust in spring wheat cultivar Alpowa. Theor. Appl. Genet. 114:1277-1287.

12. Line, R. 2002. Stripe rust of wheat and barley in North America: A retrospective historical review. Annu. Rev. Phytopathol. 40:75-118.

13. Line, R. F., and Qayoum, A. 1992. Virulence, aggressiveness, evolution, and distribution of races of Puccinia striiformis (the cause of stripe rust of wheat) in North America, 1968-87. U.S. Dep. Agric. Tech. Bull. No. 1788.
14. Mallard, S., Gaudet, D., Aldeia, A., Abelard, C., Besnard, A. L., Sourdille, P., and Dedryver, F. 2005. Genetic analysis of durable resistance to yellow rust in bread wheat. Theor. Appl. Genet. 110:1401-1409.

15. McIntosh, R. A., Devos, K. M., Dubcovsky, J., Rogers, W. J., Morris, C. F., Appels, R., and Anderson, O. A. 2005. Catalogue of gene symbols for wheat: 2005 supplement (online). Graingenes: A database for Triticeae and Avena. Published online by the U.S. Dep. Agric. http://www. wheat.pw.usda.gov/ggpages/pubs.html.

16. Pestsova, E., Ganal, M. W., and Röder, M. S. 2000. Isolation and mapping of microsatellite markers specific for D genome of bread wheat. Genome 43:689-697.

17. Qayoum, A., and Line, R. F. 1985. High-temperature, adult-plant resistance to stripe rust of wheat. Phytopathology 75:1121-1125.

18. Ramburan, V. P., Pretorius, Z. A., Louw, J. H., Boyd, L. A., Smith, P. H., Boshoff, W. H. P., and Prins, R. 2004. A genetic analysis of adult plant resistance to stripe rust in the wheat cultivar Kariega. Theor. Appl. Genet. 108:1426-1433.

19. Rogers, S. O., and Bendich, A. J. 1985. Extraction of DNA from milligram amounts of fresh, herbarium and mummified plant tissues. Plant Mol. Biol. 5:69-76.

20. Röder, M. S., Korzun, V., Wendehake, K., Plaschke, J., Tixier, M. H., Leroy, P., and Ganal, M. W. 1998. A microsatellite map of wheat. Genetics 149:2007-2023.

21. Santra, D. K., Santra, M., Uauy, C., Campbell, K. G., Chen, X. M., Dubcovsky, J., and Kidwell, K. K. 2006. Identifying QTL for hightemperature adult-plant resistance to stripe rust in wheat (Triticum aestivum L.). Page 179 in Abstracts of Plant \& Animal genome XIV.

22. SAS Institute. 1991. SAS Language and Procedures: Usage 2, Version 6, 1st ed. SAS Institute Inc., Cary, NC.

23. Somers, D. J., Isaac, P., and Edwards, K. 2004. A high-density microsatellite consensus map for bread wheat (Triticum aestium L.). Theor. Appl. Genet. 109:1105-1114.

24. Song, Q. J., Ficus, E. W., and Cregan, P. B. 2002. Characterization of trinucleotide SSR motifs in wheat. Theor. Appl. Genet. 104:286-293.

25. Stubbs, R. W. 1988. Pathogenicity analysis of yellow (stripe) rust of wheat and its significance in a global context. Pages 23-38 in: Breeding Strategy for Resistance to the Rusts of Wheat. N. W. Simmonds and S. Rajaram, eds. CIMMYT, Mexico DF.

26. Uauy, C., Brevis, J. C., Chen, X. M., Khan, I., Jackson, L., Chicaiza, O., Distelfeld, A., Fahima, T., and Dubcovsky, J. 2005. High-temperature adult-plant (HTAP) stripe rust resistance gene Yr36 from Triticum turgidum ssp. dicoccoides is closely linked to the grain protein content locus $G p c-B 1$. Theor. Appl. Genet. 112:97-105.

27. Wan, A., Zhao, Z., Chen, X., He, Z., Jin, S., Jia, Q., Yao, G., Yang, J., Wang, B., Li, G., Bi, Y., and Yuan, Z. 2004. Wheat stripe rust epidemic and virulence of Puccinia striiformis f. sp. tritici in China in 2002. Plant Dis. 88:896-904

28. Zadoks, J. C., Chang, T. T., and Konzak, C. F. 1974. A decimal code for the growth stages of cereals. Weed Res. 14:415-421.

29. Zhang, Z. J., Yang, G. H., Li, G. H., Jin, S. L., and Yang, X. B. 2001. Transgressive segregation, heritability, and number of genes controlling durable resistance to stripe rust in one Chinese and two Italian wheat cultivars. Phytopathology 91:680-686. 